\title{
ACRYLONITRILE BLOCK AND GRAFT COPOLYMERS
}

\author{
A. D. JENKINS \\ School of Molecular Sciences, University of Sussex, Brighton, BN19QJ,UK
}

\begin{abstract}
This paper presents a review of the methods available for preparing block and graft copolymers in which one component is acrylonitrile. Methods using polymer ions and polymer radicals are considered together with a useful new technique based on reactions of nitrile groups with amide compounds of group IV transition metals.
\end{abstract}

Because of its polarity and its unusual solubility properties, a polyacrylonitrile component adds great potential interest to a block or graft copolymer system and many reports can be found in the literature of attempts to prepare such copolymers. The methods used embrace a wide range of techniques; some are purely chemical, ${ }^{1-3}$ others involve ultrasonics ${ }^{4}$ or high energy radiation. ${ }^{5}$ My main purpose in this paper is to discuss three of the purely chemical methods which have developed significantly in recent years, and the methods in question involve polymer ions, polymer radicals or organometallic polymer derivatives as key reagents in the formation of the block or graft copolymer.

\section{METHODS USING POLYMER IONS}

The living polymer systems described by Szwarc $^{6}$ provide the best-known technique for preparing block copolymers but not much is recorded in the literature concerning molecules containing acrylonitrile as one component. The use of acrylonitrile as the first-phase monomer with either lithium butyl of sodium naphthalene seems to give insoluble polymers of little use, ${ }^{7}$ no doubt due to reactions at the $\mathrm{C} \equiv \mathrm{N}$ as well as the $\mathrm{C}=\mathrm{C}$ bonds, but some success has been reported with acrylonitrile as the second-phase monomer, especially in conjunction with styrene.

Claes and Smets ${ }^{8}$ were able to obtain block copolymers in this way free from homopolyacrylonitrile but much homopolystyrene was present, although this was readily removed by solvent extraction. Seymour et al..$^{7}$ have reported success with a similar system but few details are given about the need to separate the block copolymer. Methyl methacrylate has also been used as the first-phase monomer but evidently it is less satisfactory: Seymour et al. found that the product after adding acrylonitrile contained less than $10 \%$ methyl methacrylate and was quite insoluble in acetone, the inference presumably being that a transfer mechanism was responsible for the formation of much homopolyacrylonitrile.

Block copolymers of styrene and acrylonitrile were prepared by Perry ${ }^{9}$ using a different technique. He made polystyrene in a free radical system with molecular growth controlled by transfer to dibutylphosphine so that he obtained an initial $\overline{\mathrm{P}}$ of about 400 with $95 \%$ of the polystyrene chains containing a phosphine residue at one end. Since dialkyl phosphines initiate the anionic polymerization of acrylonitrile, the first-phase polymer is ideally suited to form the basis of block copolymer and acrylonitrile contents in the range $14-76 \%$ were obtained. Extraction techniques established a fairly high purity level for the block copolymers thus obtained.

\section{METHODS USING POLYMER RADICALS}

During the investigation of the bulk polymerization of acrylonitrile it was deduced from kinetic observations that under certain conditions a significant, or even substantial, fraction of the radicals generated remain occluded in the precipitated polymer for long periods of time. ${ }^{10,11}$ At temperatures as high as $60^{\circ} \mathrm{C}$ occlusion affects the velocity constant for the termination process but is not severe enough to trap radicals; at $25^{\circ} \mathrm{C}$, on the other hand, it has been estimated that perhaps $1 \%$ of the radicals generated in photopolymerization become so tightly embedded in the polymer that they are unable to propagate further, although they are liberated by raising the temperature.

Very fine control is possible in this situation because elevation of the temperature to $40^{\circ} \mathrm{C}$ facilitates propagation but does not sufficiently open the structure that the occluded radicals terminate in appreciable numbers, whereas heating to $60^{\circ} \mathrm{C}$ destroys them all in a short time. The environment of the trapped radicals can be changed by replacement of the residual acrylonitrile by another liquid, and if this contains a monomer the liberated radicals will react with it to produce block copolymer. ${ }^{12}$ The precise nature of the block copolymer will then depend upon the mechanism which terminates the new growth, disproportionation or transfer giving an $\mathrm{AB}$ type and combination an $\mathrm{ABA}$ type of block structure.

The type of system discussed here was first described by Bamford and Jenkins, ${ }^{12}$ who demonstrated that the nature of the second monomer had a large influence on the extent of second-phase polymer formed. This is, of course, not surprising since the system must be very sensitive to the degree of interaction between the liquid and the polymer, a point taken up later by Seymour and his colleagues. ${ }^{13}$ Among the systems examined by Bamford and Jenkins, ${ }^{12}$ the one which gave the greatest amount of polymer was polyacrylonitrile/methyl acrylate; it was possible here to attain almost complete conversion of the methyl acrylate to polymer because the rate of radical destruction is very low.

This observation highlights a key factor in the situation, viz. that if polymer and liquid interact too weakly the occluded radicals will not be liberated enough to propagate, while swelling on too large a scale will allow the radicals to terminate too rapidly to produce much block copolymer. In the former case a remedy is at hand if a small proportion of swelling agent, such as dimethylformamide, is added to the second-phase monomer; the latter problem can possibly be mitigated by mixing the monomer with a liquid which has very little interaction with polymer or by lowering the temperature, but these 
factors also tend to limit the extent of polymerization.

The original work was followed up by Hiemeleers and Smets $^{14}$ who showed that the molar uptake of second monomer by polyacrylonitrile radicals was below $5 \%$ with styrene, vinyl acetate, acrylamide and vinylidene chloride, a little higher with methyl methacrylate (9\%) and more substantial with methyl acrylate in dioxane (14\%) and acrylic acid (16\%) but that none of these monomers challenged acrylonitrile itself (36\%). Thus bulk methyl acrylate seems to be an exceptionally favourable case. These authors made the helpful observation that only very small quantities of second step homopolymer are formed in the process, but of course a drawback to this method of preparing block copolymers is the homopolyacrylonitrile, from association with which the block copolymer requires to be extracted.

Other heterogeneous polymerizations than that of acrylonitrile were tried as the basis of this method of block copolymer formation, ${ }^{14}$ the photopolymerization being carried out at $25^{\circ} \mathrm{C}$. With polyacrylamide only small amounts of second monomer were observed to become attached and with polyvinylchloride none. This confirms the general belief that trapped radicals are not obtained in the bulk vinyl chloride system as they are with acrylonitrile at ambient temperature.

A further advance was made by Minoura and Ogata ${ }^{15}$ who had access to lower temperatures for the polymerization of the first monomer through use of the $\mathrm{Bu}_{3} \mathrm{~B} / \mathrm{O}_{2}$ system as initiator. They used acrylonitrile in THF solution at $0^{\circ} \mathrm{C}$ and vinyl chloride in methanol at $-78^{\circ} \mathrm{C}$, among other reaction conditions. It seems that the first-phase polymer could be handled in air without impairment of activity.

These authors demonstrated that the addition of swelling agent was useful, thus the amount of methyl methacrylate which could be caused to add to polyacrylonitrile radicals was more than doubled by admixture with roughly its own volume of dimethylformamide. A similar but less spectacular result was attained with vinyl acetate.

A surprising feature of this work is the very slow rate of addition in the second phase, even at temperatures as high as $80^{\circ} \mathrm{C}$, but presumably the degree of swelling of polyacrylonitrile in, say, styrene is so low that the occlusion barrier is only marginally reduced.

Methyl methacrylate has also been reported as a second-phase monomer in admixture with solvent, by Yoshimoto et $a l^{16}$

The value of the presence of swelling agent in the second-phase medium was confirmed by Seymour et al. ${ }^{7}$ who showed that about $5 \%$ of dimethylformamide (based on the amount of first-phase polymer) was sufficient to raise the yield of second-phase polystyrene by a factor of four. Up to above five times this quantity of dimethylformamide produced the same result but larger quantities were conducive to progressively lower yields, no doubt because excessive swelling is responsible for termination.

Acrylonitrile has also been used as the second-phase monomer when other heterogeneous polymerizations have been employed in the first phase, as reported by Seymour and his team. The first phase in these cases has been variously, styrene-maleic anhydride copolymer, ${ }^{17}$ styrene ${ }^{18}$ or methyl methacrylate ${ }^{18}$ the polymerization in each case being carried out in conditions selected to cause polymer precipitation. In the case of methyl methacrylate, hexane and 1-propanol proved to be useful solvents for the monomer and they provided media in which the polymerization was much more rapid than in a good solvent, such as benzene. Seymour reports that stable radicals are not obtained in these systems above the glass transition temperature (presumably the $\mathrm{Tg}$ measured in the particular system rather than that of the dry polymer).

Seymour has placed considerable emphasis on the importance of the relationship between solubility parameters of the first-phase polymer and the second-phase medium. While it is true that such a correlation does exist, so that a difference of less than $3 \cdot 1$ Hildebrand units is necessary for penetration of polymer by liquid to recur, ${ }^{13}$ the original observations on acrylonitrile polymerization ${ }^{11}$ indicate that temperature could also be a crucial factor but there does not seem to be a sufficient body of systematic data at the present time to test this possibility.

More recently Seymour et al. ${ }^{19}$ have reported that the addition of fumed silica to the first-phase reaction mixture facilitates the preparation of block copolymers.

\section{METHODS USING ORGANOMETALLIC POLYMER DERIVATIVES}

In recent years we have studied a new system for the polymerization of acrylonitrile $\mathrm{e}^{20-24}$ which affords a useful method of preparing both block and graft copolymers with acrylonitrile as the second phase. A brief description of this system will be presented as a basis for discussing the preparation of the binary molecules.

Compounds containing bonds between group IV transition metals and nitrogen act as very efficient and specific initiators for the polymerization of monomers containing $\mathrm{CN}$ groups, that is, for acrylonitrile and methacrylonitrile. The former monomer is much more active than the latter but both respond to the initiators which themselves increase markedly in activity from Ti-N to Zr-N. Most of our work has been carried out with $\mathrm{Ti}-\left(\mathrm{NMe}_{2}\right)_{4}$, and this compound, designated $\mathrm{T}_{4}$, will figure in the summary below.

Polymerization of acrylonitrile takes place smoothly when the monomer is mixed with $\mathrm{T}_{4}$ in a simple hydrocarbon solvent at room temperature. It is necessary to dry all reagents thoroughly and to exclude oxygen but a nitrogen blanket is adequate for the latter purpose. The solvent is preferably methyl cyclohexane because the low freezing point of this substance affords a large liquid range over which to study the influence of temperature. Using concentrations of $\left[\mathrm{T}_{4}\right]_{0} \sim 10^{-3} \mathrm{M}$ and $[\mathrm{AN}]_{0} \sim 1 \mathrm{M}$ polymerization reaches $50 \%$ conversion in less than $50 \mathrm{~min}$ at $25^{\circ} \mathrm{C}$.

Extensive studies of the reaction mechanism have led to the conclusion that the reaction follows the following sequence:

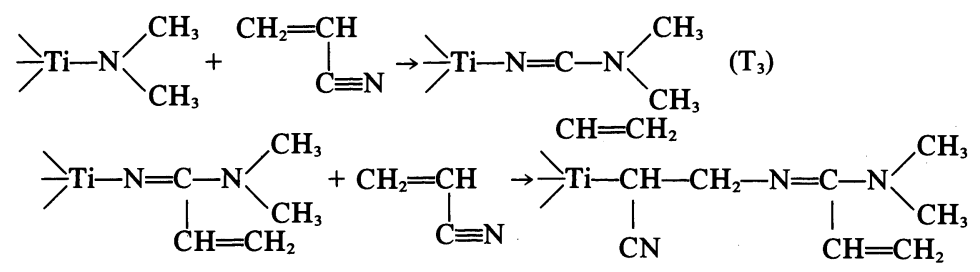




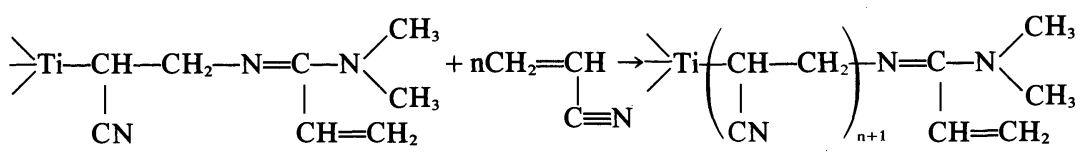

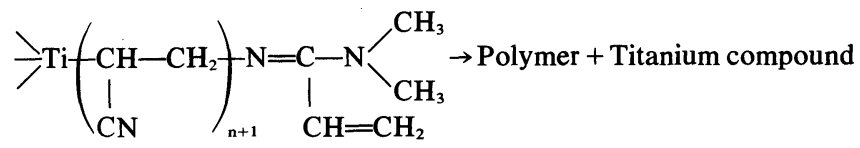

An alternative formulation of step (1) is<smiles></smiles>

but in the present context it does not matter whether the monomer reacts in the 1,4 mode or at the $-\mathrm{CN}$ group alone.

The evidence for this mechanism has been discussed in some detail elsewhere ${ }^{21,22}$ and it seems that the equations above represent the essential steps in the process. Of course, since the initiator $\mathrm{T}_{4}$ contains four Ti-N bonds there may be multiple activity at the transition metal centre, indeed it is clear that such is the case.

Some very useful information has been derived from the use of propionitrile as a model substance which should be able to undergo only step (1) of the sequence shown but a multiple number of times up to a maximum of four. NMR studies show clearly that the full sequence of four insertions occurs and, if this possibility is also admitted for the monomer, a full kinetic scheme can be set up and solved by computer methods to furnish rate constants for the various steps. ${ }^{23}$

The first step in the reaction between $\mathrm{T}_{4}$ and propionitrile will give the compound

$$
\left.\left(\begin{array}{l}
\mathrm{CH}_{3} \\
\mathrm{CH}_{3}{ }^{\prime}
\end{array}\right)^{\mathrm{N}}\right)_{-\mathrm{Ti}-\mathrm{N}=\mathrm{C}-\mathrm{N}} \stackrel{\mathrm{N}}{\mathrm{CH}_{2} \mathrm{CH}_{3}}{ }_{\mathrm{CH}_{3}}^{\mathrm{CH}_{3}}
$$

which is the propionitrile analogue of compound $\mathrm{T}_{3}$ in the reaction scheme. It would seem entirely possible that this compound should also be able to promote step (2) by reaction with acrylonitrile monomer, and such is indeed the case. If $T_{4}$ and propionitrile are mixed together and allowed to react for a few hours the mixture proves to be a highly potent initiator of acrylonitrile polymerization, in fact it is an easy matter to achieve $50 \%$ polymerization in about $1 \mathrm{~min}$. This observation is the key to the method we have used to adapt the $T_{4}$ type of initiation to the preparation of block and graft copolymers by using nitrile groups attached to polymers as analogues of the nitrile groups in propionitrile for the initiation of acrylonitrile or methacrylonitrile polymerization. ${ }^{25}$ This can best be explained by means of two typical examples.

\section{(i) Graft copolymer}

First a random copolymer of acrylonitrile and styrene was prepared by the standard radical technique. This provides a polymer with essentially a styrene-type backbone furnished with a few nitrile groups at random points. This copolymer was regarded as a high molecular weight saturated nitrile, parallel to propionitrile, and was allowed to react with $T_{4}$ for a few days to encourage all the nitrile groups to undergo step (1) of the reaction scheme. The polymer was then carefully and thoroughly washed to remove the excess $T_{4}$ completely. When this process was complete acrylonitrile was added and the mixture left to react so that acrylonitrile grafts could grow on the polystyrene backbone. In this way it proved possible to add grafts of polyacrylonitrile equal in total weight to that of the polystyrene backbone. Since the molecular weights of styrene and acrylonitrile differ by almost exactly a factor of two this means that the final polymer was roughly $2: 1$ acrylonitrile: styrene on a molar basis.

\section{(ii) Block copolymer}

Polystyrene was prepared using azo-bisisobutyronitrile as initiator under conditions where very little transfer occurred and termination of molecular growth was consequently largely the result of radical combination reactions. The nitrile groups in the initiator fragments, one at each end of the polystyrene molecule, provide suitable points for attachment of polyacrylonitrile blocks after treatment with $T_{4}$. Once again, it proved possible to add a roughly equal weight of polyacrylonitrile to the original polystyrene backbone.

In all these reactions it is necessary to carry out careful checks for the presence of homo-polyacrylonitrile and to prove that the structure of the final polymer is that expected from the premises described above. According to our experiments, the reactions proceed as expected with remarkable efficiency so we conclude that the $T_{4}$ technique provides an extremely satisfactory route to block and graft copolymers in which acrylonitrile is the second-phase monomer.

\section{REFERENCES}

${ }^{1}$ C. H. Bamford and E. F. T. White, Trans. Faraday Soc. 54, 268 (1958).

${ }^{2}$ S. Aoki, T. Otsu and M. Imoto, Kogyo Kagaku Zasshi, 67(6), 971 (1964).

${ }^{3}$ R. B. Seymour, D. R. Owen, M. L. McGee, C. J. Losada and M. Clark, Prep. Amer. Chem. Soc. ORPL Div. 33(1), 663 (1973).

${ }^{4}$ A. Henglein, Makromol. Chem. 14, 128 (1954).

${ }^{5}$ A. Chapiro, J. Polym. Sci. 23, 377 (1957).

${ }^{6} \mathrm{M}$. Szwarc. Carbanions, Living Polymers and Electron Transfer Processes. Interscience, New York (1968).

${ }^{7}$ R. B. Seymour, D. R. Owen, G. A. Stahl, H. Wood and W. N. Tinnerman, Prep. Amer. Chem. Soc. POLY Div., 14(2), 658 (1973). Appl. Polym. Symposium, 25, 69 (1974).

${ }^{8}$ P. Claes and G. Smets, Makromol. Chem. 44-46, 212 (1961).

${ }^{9}$ E. Perry, J. Appl. Polymer Sci. 8, 2605 (1964).

${ }^{10}$ C. H. Bamford and A. D. Jenkins, Proc. Roy. Soc. A, 216, 515 (1953).

${ }^{11}$ A. D. Jenkins, in Vinyl Polymerization. (G. E. Ham, ed), Chapt. 6 (1967).

${ }^{12}$ C. H. Bamford and A. D. Jenkins, J. Chim. Phys. 56, 798 (1959). 
${ }^{13}$ R. B. Seymour, P. D. Kincaid and D. R. Owen, Prep. Amer. Chem. Soc. POLY Div. 13(1), 522 (1972).

${ }^{14} \mathrm{~J}$. Hiemeleers and G. Smets, Makromol. Chem. 47, 7 (1961).

${ }^{15}$ Y. Minoura and Y. Ogata, J. Polym. Sci. (A-1), 7, 2547 (1964).

${ }^{16} \mathrm{~K}$. Yoshimoto, S. Yamamoto and M. Oiwa, Kogyo Kagaku Zasshi, 73, 1177 (1970).

${ }^{17}$ R. B. Seymour, P. D. Kincaid and D. R. Owen, J. Paint Tech. 45, 59 (1973).

${ }^{18}$ R. B. Seymour, D. R. Owen and G. A. Stahl, Prep. Amer. Chem. Soc. ORPL Div. 33(1), 670 (1973).

${ }^{19}$ R. B. Seymour, G. A. Stahl and H. Wood, Prep. Amer. Chem. Soc. POLY Div. 16(1), 470 (1975).
${ }^{20}$ A. D. Jenkins, M. F. Lappert and R. C. Srivastava, J. Polym. Sci. (B), 6, 865 (1968).

${ }^{21}$ A. D. Jenkins, M. F. Lappert and R. C. Srivastava, Europ. Polym. J. 7, 289 (1971).

${ }^{22}$ N. C. Billingham, L. M. Boxall and A. D. Jenkins, Europ. Polym. J. 8, 1045 (1972)

${ }^{23}$ N. C. Billingham, L. M. Boxall, A. D. Jenkins and P. D. Lees, Europ. Polym. J. 10, 981 (1974).

${ }^{24}$ N. C. Billingham, L. M. Boxall, A. D. Jenkins and P. D. Lees, Europ. Polym. J. 10, 991 (1974).

${ }^{25} \mathrm{~N}$. C. Billingham, A. Gungor and A. D. Jenkins. To be published. 